\title{
Endoscopic Ultrasound Guided Fine Needle Aspiration in the Diagnosis of Intra-abdominal Lesions
}

\author{
Alam AHMT*, Chowdhury MAK, Das CR \\ Department of Surgery, Bangabandhu Sheikh Mujib Medical University, Dhaka, Bangladesh
}

\begin{abstract}
Background: Endoscopic ultrasound guided fine-needle aspiration cytology (EUS-FNA) in the diagnosis of intra-abdominal lesion is a safe and accurate procedure which is practiced in various parts of the world. However, this technique is very new in Bangladesh.

Objective: This study was aimed to evaluate the diagnostic utility of endoscopic ultrasound guided fine needle aspiration (EUS-FNA) in the diagnosis of abdominal lesions.

Methods: EUS-FNA was carried out on a total of 48 cases during the study period. The lesions were categorized according to the site of FNA. Clinical impression was compared with the final cytological diagnosis and the percentage of non diagnostic smears was calculated.

Results: Out of 48 cases, 10 cases (20.8\%) were reported as inconclusive while a definite diagnosis was given in 38 cases $(79.21 \%)$. The mean patient age was 47.68 years. There were $28(58.33 \%)$ males and $20(41.67 \%)$ females. The most common site biopsied was pancreas followed by abdominal lymph node. The average number of passes made was two.
\end{abstract}

Conclusion: Endoscopic Ultrasound Guided Fine-Needle Aspiration in the diagnosis of intra-abdominal lesion is a useful procedure in the evaluation of deep seated lesions of gastrointestinal tract (GIT) and abdominal cavity.

Keywords: Endoscopic ultrasound, Fine needle aspiration cytology, Intra-abdominal lesions, Biopsy

\section{Introduction}

A wide variety of inflammatory and neoplastic lesions can occur in the abdominal cavities. They are detected as space occupying lesion by Ultrasonography, Endoscopy or by C.T Scan. ${ }^{1-3}$ However these imaging techniques are not useful in the exact diagnosis of the pathologic process or differentiation between inflammatory process, benign and malignant lesions. ${ }^{4-6}$

The development of endoscopic ultrasound scanning (EUS) began in the early 1980s with mechanical radial scanning transducers. ${ }^{1}$ In spite of an excellent imaging resolution the method did not gain widespread popularity until the development of EUS-guided fine needle aspiration biopsy (EUS-FNA). ${ }^{2}$ EUS-FNA is at present performed on a routine basis at many endoscopic centers and it is evident that this procedure has a

*Correspondence: AHM Towhidul Alam, Department of Surgery, Bangabandhu Sheikh Mujib Medical University, Dhaka, Bangladesh; e-mail: gsdbsmmu@yahoo.com ORCID: https://orcid.org/0000-0002-4355-3516 major impact on the therapeutic management of patients by obtaining a definite tissue diagnosis from lesions outlined by endosonography. ${ }^{3,4}$

Endoscopic ultrasound (EUS) which involves fiberoptic endoscopy with endoluminal ultrasound; when combined with needle aspiration of the lesions through the endoscope can lead to direct sampling of the lesion leading to definite diagnosis. ${ }^{7-11}$ Endoscopic ultrasound guided fineneedle aspiration cytology (EUS-FNA) in the diagnosis of intra-abdominal lesion is a safe and the accurate procedure which is practiced in various parts of the world. ${ }^{12}$ However, this technique is very new in Bangladesh. It was started at Department of Surgery, BSMMU in 2015 and since then has gained much popularity.

Previous studies have examined endoscopic ultrasound with fine needle aspiration (EUS-FNA) as a safe and effective method to obtain tissue diagnosis in these cases. ${ }^{1,2}$ Initially, it was postulated that endosonographic features alone 
could predict malignant potential of lymph nodes, but later work has shown that fine needle aspiration adds to diagnostic yield and accuracy, potentially precluding the need for surgical exploration. ${ }^{3-6}$ EUS-FNA has repeatedly been proven a safe exam with examination with minimal complication rates and sensitivities and specificities approaching $100 \% ., 7-8$

There is also considerable evidence that EUS-FNA in experienced hands can replace many other far more invasive and risky diagnostic procedures, such as, diagnostic laparoscopy and even laparotomy ${ }^{6,7}$ However, the technique is not easy to master and considerable energy and effort has to be invested before the practitioner reaches an acceptable success rate.

In this study, it was evaluated the diagnostic yield of endoscopic ultrasound guided cytology of abdominal lesions.

\section{Material and Methods}

It was a prospective observational study carried out in the department of surgery BSMMU from June 2015 to August 2016. This study was conducted to evaluate the diagnostic utility of endoscopic ultrasound guided fine needle aspiration (EUSFNA) in the diagnosis of abdominal lesions. The patients presented with a space occupying lesion, a mass or abdominal lymphadenopathy detected by abdominal ultrasound and CT scan of abdomen were included. Those patients with a previous history of malignancies were excluded. Total 48 cases were included in this study. EUS was performed both on outpatient basis and also on admitted patient. The patients were placed in left lateral decubitus position and under total intravenous anesthesia (TIVA) a linear echoendoscope was used to evaluate the abdominal lesions. After the lesion was identified, colour flow and doppler sonography were performed to exclude intervening vascular structures, vascular lesions and to choose a vessel free needle tract.

It is obvious that EUS-FNA is not at all limited to gastroenterology, as the gastrointestinal tract traverses through anatomical regions related to other medical specialties such as pulmonology, thoracic surgery, internal medicine, oncology, urology, gynecology and endocrinology. ${ }^{5}$ There is also considerable evidence that EUS-FNA in experienced hands can replace many other far more invasive and risky diagnostic procedures, such as mediastinoscopy, diagnostic laparoscopy and even laparotomy or thoracotomy. ${ }^{6,7}$ However, the technique is not easy to master and considerable energy and effort has to be invested before the practitioner reaches an acceptable success rate. This is reported in the literature to be about $90-95 \%$, with an overall sensitivity and specificity of $90 \%$ and $100 \%$, respectively. 8 $14 \mathrm{~mm}$ may be imaged and consequently biopsied (Even minute lesions down to a size of $51 \mathrm{~mm}$ or large $\leq 25$ ). Moreover, contrary to what should be expected, the size of the lesions (small $>\mathrm{mm}$ ) does not necessarily influence the overall diagnostic yield, sensitivity, specificity or accuracy of the method. Consequently, the accuracy of the method is variable in the literature between 85 and $95 \%$, even in the cases where other alternative biopsy techniques have failed. ${ }^{8-15}$

All the FNAs were carried out using 22 gauge needle equipped with a stylet that had a tight fit within the needle to minimize contamination by the gastrointestinal tract (GIT). The catheter that contained the needle was introduced through the working channel of the Endoscope. When the Tip of the catheter was visualized, the needle was advanced from the catheter sheath, through the wall of the bowel into the target lesion under ultrasound guidance. The stylet was removed when needle was within the target lesion and aspiration biopsy was performed by moving the needle back and forth for 5-10 seconds. The needle was then retracted. In few cases suction was applied during the biopsy. If additional passes were needed, the stylet was reinserted into the needle and the steps were repeated. This procedure was stopped after confirmation of adequacy of material obtained by on site examination of the smears to see the adequate cellular materials in the slide.

The aspirate was placed on glass slide and two to three smears were prepared from a single aspirate by placing another slide over it and spreading the aspirate on the slides. Both air dried and alcohol fixed smears were prepared. Wet fixed smears were placed in alcohol of difference concentration. Air dried smears were stained with Diff-Quik stain and reviewed immediately to confirm the adequacy of the material. These slides were then submitted at the department of pathology, BSMMU along with a proforma 
containing clinical details, EUS findings and provisional clinical diagnosis.

The cases were evaluated for adequacy, site of biopsy, number of passes, clinical impression, provisional and final cytological diagnosis. The term inadequate or non-diagnostic aspirate was reserved for those smears in which the cellularity was not sufficient to characterise the lesion.

\section{Results}

During the year June 2015 to August 2016, 48 cases of EUS-FNA were sent to department of Pathology, BSMMU. Twenty eight patients were male and 24 were female. Their ages ranged from 14 to 77 years and the mean age was 47.86 years.

The most common site of FNA was pancreas constituting 28 (58.3\%), followed by lymph nodes $14(29.2 \%)$ cases. Other site includes stomach, duodenum \& suprarenal glands (figure 1).

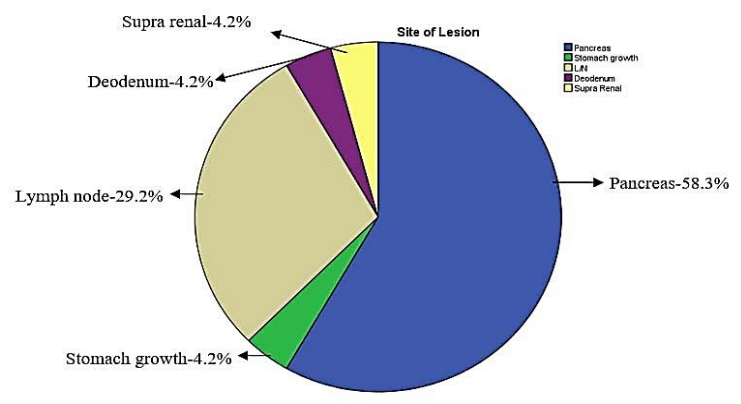

Figure 1: Site wise distribution of EUS-FNA.

The average number of passes made was 2 with a minimum of 1 pass and maximum of 4 passes. The size of the lesion was documented in 43 cases. The average size of the lesions was $3 \mathrm{~cm}$. with a minimum of $1 \mathrm{~cm}$. and maximum of $10 \mathrm{~cm}$. Out of 48 cases, 10 were reported as inadequate. Out of the remaining 38 cases, 29 (60.4\%) were reported as neoplastic lesions (table I).

Table I: Frequency distribution of neoplastic lesions

\begin{tabular}{lcc}
\hline & Frequency & Percent \\
\hline Adenocarcinoma & 6 & 12.5 \\
Positive for malignancy & 7 & 14.6 \\
Non-hodgkins lymphoma & 6 & 12.5 \\
Neuro-Endocrine neoplasm & 1 & 2.1 \\
Metastatic carcinoma & 4 & 8.3 \\
GIST & 1 & 2.1 \\
Solid pseudopapillary tumour & 3 & 6.3 \\
lymphoma & 1 & 2.1 \\
Total & 29 & 60.4 \\
\hline
\end{tabular}

The most common being adenocarcinoma and non-hodgkins lymphoma both of them were 6 in number followed by undetermined malignancy in 7 cases, solid pseudopapillay tumor in 3 cases, metastatic carcinoma in 4 cases, GIST in 2 cases, endocrine neoplasm in one case.

Nine $(18.8 \%)$ lesions were reported as benign nonneoplastic in nature. Seven of them were normal, one of the non-neoplastic lesions was chronic granulomatous inflammation in suprarenal gland and rest of the case was a inflammatory lesion in pancreatic head. (table II)

Table II: Frequencies of non-neoplastic lesions

\begin{tabular}{lcc}
\hline & Frequency & Percent \\
\hline Normal & 7 & 14.6 \\
Granulomatous Lesion & 1 & 2.1 \\
Inflammatory & 1 & 2.1 \\
Total & 9 & 18.8 \\
\hline
\end{tabular}

Of 14 abdominal lymph nodes sampled, nonhodgkins lymphoma was diagnosed in 4 lymph nodes followed by metastatic carcinoma in 4 cases (table III).

Table III: Final diagnosis of lymph node lesion with FNA.

\begin{tabular}{lcc}
\hline & Frequency & Percent \\
\hline Normal & 2 & 4.2 \\
Metastatic carcinoma & 4 & 8.3 \\
Non-hodgkins Lymphoma & 4 & 8.3 \\
Blood only & 4 & 8.3 \\
Total & 14 & 29.2 \\
\hline
\end{tabular}

Sixteen cases of neoplastic lesion were diagnosed in total of 28 pancreatic aspirates (table IV).

Table IV: Final diagnosis of pancreatic lesion with FNA.

\begin{tabular}{lcc}
\hline & Frequency & Percent \\
\hline Normal & 3 & 6.3 \\
Adenocarcinoma & 6 & 12.5 \\
Solid Pseudopapillary Tumour & 3 & 6.3 \\
Neuroendocrine Tumour & 1 & 2.1 \\
Positive for Malignancy & 4 & 8.3 \\
Blood Only & 6 & 12.5 \\
Non-Hodgkins Lymphoma & 1 & 2.1 \\
Lymphoma & 1 & 2.1 \\
Inflammatory & 1 & 2.1 \\
Cyst Adenoma & 2 & 4.2 \\
Total & 28 & 58.3 \\
\hline
\end{tabular}

No complication was observed following EUSFNA in any case except minimal discomfort at the time of needle puncture. 


\section{Discussion}

Endoscopic ultrasound (EUS) is a rapid, safe, economical and accurate diagnostic procedure that can be used in the assessment of various neoplastic and non- neoplastic diseases involving gastrointestinal tract \& abdominal cavity. ${ }^{13}$ For gastrointestinal tract lesions, EUS is particularly helpful in identifying the origin of the lesion whether it is arising from the wall or is extrinsic and compressing the lumen from outside. ${ }^{14-17}$ EUS can also identify the layer of the bowel wall from which the lesion arises and it also provides information on the extent and borders of the lesion. However, definitive differentiation between benign and malignant lesions using EUS alone is usually not possible. Therefore tissue sampling for EUS-FNA is required to establish a conclusive diagnosis.

Advantages of the technique include its utility in patients with inoperable lesions, and in whom surgery is contraindicated due to some reason such as fitness for anaesthesia. With diagnoses like tuberculosis, inflammatory lesion, lymphoma etc treatment can be started on the basis of cytological diagnosis. ${ }^{18-20}$ There are few limitations including very small lesions, inaccessible lesions, vascular lesions, calcifications, cavitating and necrotic lesions.

As the diagnosis is rapidly available on EUSFNA, appropriate medical or surgical therapy can also be started earlier, at times avoiding unnecessary and often invasive diagnostic surgical procedures. In lesions requiring surgical procedures, operative time is reduced as intraoperative evaluation is not required.

Moreover, as this procedure is performed on an outpatient basis, surgical mortality, morbidity and patient hospitalization is reduced thereby benefiting the patient and health care system. In our study the diagnostic yield was $79 \%$ with non diagnostic aspirates yielded in 10 cases. The factors contributing to inadequate or non diagnostic aspirates include very small lesion, necrotic centre of the lesion, sampling error leading to tissue sampling from the inappropriate site, haemorrhagic lesions and cytological error due to poor sample quality.
It is also important to note that in this study, the most common site of EUS-FNA was pancreas followed by lymph node and Sampling of pancreatic lesions is very difficult. EUS-FNA thus provides an alternate safe, rapid and non invasive method of sampling these lesions. ${ }^{21-25}$

Another advantage of EUS-FNA is that it is very effective method for detecting and sampling very small lesions of pancreas so that early diagnosis is possible in pancreatic neoplasms. It has been shown that EUS alone is more sensitive than CT scan and magnetic resonance imaging in detecting very small lesions of pancreas, especially when they are smaller than $3 \mathrm{~cm} .{ }^{26}$ In this study also, the mean size of lesion was $3 \mathrm{~cm}$ with a minimum of one $\mathrm{cm}$. sampled from pancreatic lesion. The use of stylet during advancement of the biopsy needle through the bowel wall minimizes the plugging of the needle tip with GI tract epithelium.

However, this process is sometimes cumbersome because of the inaccessibility of deepseated lymph nodes or organs (e.g., pancreas) and due to risk of complications with the percutaneous approach. ${ }^{2}$ Achieving sufficient tissue is crucial. Therefore, invasive and costly procedures, such as thoracotomy, laparotomy, mediastinoscopy, or laparoscopy, may be required.

EUS-guided fine needle aspiration cytology (EUSFNAC) and biopsy (EUS-FNAB) are excellent techniques for obtaining adequate materials for cytological or histological diagnosis of various lesions. ${ }^{3,4}$ The advantages of EUS over other imaging techniques include real-time puncture, reduced risk of complications due to the proximity of the needle to the lesion, and the ability to sample small lesions that might be hard to sample using other methods. Finally, EUS allows access to deep-seated lesions, which is a challenge with other techniques. EUS makes sampling of mediastinal, retroperitoneal, and perigastrointestinal lymph nodes possible, with an overall accuracy between $65 \%$ and $100 \% .^{5}$ The present manuscript summarizes the available evidence related to the use of EUS-FNAC or EUSFNAB for the diagnosis of lymphoma, including the classification of lymphoma subtypes according to the most recent classification systems. In addition, it is discussed the utility of EUS-FNA in the diagnosis of primary extranodal lymphomas 
and technical issues related to optimization of tissue acquisition.

Follow-up of these patients and confirmation of our diagnoses with the histological diagnoses after surgical treatment could not be carried out because most of the patients undergoing EUSFNA came from different hospitals. Surgical treatment if rendered also took place in different set ups and all the histopathology samples were not sent to the dept. of surgery, BSMMU. The patient those who were admitted in BSMMU, most of them were inoperable and only diagnostic evaluation was done.

\section{Conclusion}

In conclusion, findings of the study suggests that EUS-FNA is a reliable and effective procedure with adequate yield in the diagnosis of neoplastic and non-neoplastic lesions of GI tract and abdominal cavity.

\section{Acknowledgement}

The authors are grateful to Bangabandhu Sheikh Mujib Medical University authority for inspiration and support to conduct the study as the research project of the university.

\section{References}

1. Fleischer AC, Muhletaler CA, James AE Jr. Sonographic assessment of the bowel wall, AJR Am J Roentgenol. 1981; 136: 887-91.

2. Morgan CL, Trought WS, Oddson TA, Clark WM, Rice RP. Ultrasound Patterns of disorders affecting the gastrointestinal tract. Radiology. 1980; 135:12935 .

3. Chhieng DC, Jhala D, Jhala N, Eltoum I, Chen VK, Vickers S, et al. Endoscopic ultrasound-guided fine needle aspiration biopsy; A study of 103 cases. Cancer. 2002; 92:232.

4. Heintz A, Mildenberger P, Georg M, Braunstein S, Junginger T. Endoscopic ultrasonography in the diagnosis of regional lymph nodes in esophageal and gastric cancer - results of studies in vitro, Endoscopy. $1993 ; 25: 231-5$

5. Kaufman AR, Sivak MV Jr. Endoscopic ultrasonography in the differential diagnosis of pancreatic disease. Gastrointest Endosc. 1989; $35: 214-9$

6. Vander Noot MR 3rd, Eloubeidi MA, Chen VK, Eltoum I, Jhala D, Jhala $\mathrm{N}$ et al. Diagnosis of gastrointestinal tract lesions by endoscopic ultrasound guided-guided fine-needle aspiration biopsy. Cancer. 2004; 102:157-63.
7. Boyce GA, Sivak MV Jr, Lavery IC, Fazio VW, Church JM, Milsom J et al. Endoscopic ultrasound in the pre-operative staging of rectal carcinoma. Gastrointest Endosc. 1992; 38:468-71.

8. Akahoshi K, Misawa T, Fujishima H, Chijiwa Y, Maruoka A, Ohkubo A et al. Preoperative evaluation of gastric cancer by endoscopic ultrasound. Gut. 1991; 32:479-82.

9. Rosch T, Lorenz R, Braig C, Feuerbach S, Siewert JR, Schusdziarra $\mathrm{V}$ et al. Endoscopic ultrasound in pancreatic tumor diagnosis Gastrointest Endosc. 1991; 37:347-52.

10. Rosch T, Lorenz R, Braig C, Classen M. Endoscopic Ultrasonography in diagnosis and staging of pancreatic and biliary tumors. Endoscopy. 1992; 24:304-8

11. Rice TW, Boyce GA, Sivak MV. Esophageal ultrasound and the preoperative staging of carcinoma of the esophagus. J Thorac Cardiovasc Surg. 1991; 101:536-43

12. Vilmann P, Saftoiu A, Endoscopic ultrasounf guided fine needle aspiration biopsy: equipment and technique, J Gastroenterol Hepatol. 2006; 26:1646-55.

13. Anand D, Barroeta JE, Gupta PK, Kochman M, Baloch ZW. Endoscopic ultrasound guided fine needle aspiration of non pancreatic lesions: an institutional experience. J Clin Pathol. 2007; 60:125462.

14. Bhutani MS, Logrono R, Endoscopic ultrasoundguided fine-needle aspiration cytology for diagnosis above and below the diaphragm, J Clin Ultrasound 2005; 33:401-11.

15. Caletti G, Odegaard S, Rosch T, Sivak MV, Tio TL, Yasuda K. Endoscopic ultrasonography (EUS): a summary of the conclusions of the Working Party for the Tenth World Congress of Gastroenterology Los Angeles, California October, 1994. The Working Group on Endoscopic Ultrasonography, Am J Gastroenterol. 1994; 89:S138-43.

16. Rowley VA, Cooperberg PL, Ultrasound guided biopsy. Interventional ultrasound, In: Clinics in "Diagnostic Ultrasound", E Vansonnenberg, editor, vol 21. Churchill Livingstone, New York. 1987; pp $59-76$

17. Jhala NC, Jhala D, Eltoum I, Vickers SM, Wilcox CM, Chhieng DC, et al. Endoscopic ultrasound guided fine needle aspiration biopsy: a powerful tool to obtain samples from small lesions. Cancer. 2004; 102:239-46.

18. Kedar RP, Patel VH, Merchant SA, Aggarwal V, Pandit AA. Ultrasound guided aspiration cytology-a valuable diagnostic aid. J Postgrad Med. 1991; 37:847.

19. Klapman JB, Logrono R, Dye CE, Waxman I. Clinical Impact of on-site cytopathology interpretation on endoscopic ultrasound - guided fine needle aspiration. Am J Gastroenterol. 2003; 98:1289-94.

20. Wiersema KJ, Wiersema LM, Khusro Q, Cramer HM, Tao LC. Combined endosonography and fine-needle 
aspiration cytology in the evaluation of gastrointesinal leisons. Gastrointest Endosc. 1994; 40:199-20.

21. Islam T, Hossain F, Rumpa et al. Ultrasound guided fine needle aspiration cytology: a sensitive diagnostic tool for diagnosis of intra-abdominal lesions. BMRC Bulletin 2013; 39:14-17.

22. J-M Dumonceau et al. Indications, results and clinical impact of endoscopic ultrasound (EUS)- guided sampling in gastroenterology: European Society of Gastrointestinal Endoscopy (ESGE) Clinical Guideline. Endoscopy. 2011; 43:897-909.

23. Rajesh Puri et al. Take-home message, Diagnostic yield of EUS-guided FNA and cytology in suspected tubercular intra-abdominal lymphadenopathy. Gastrointestinal Endoscopy. 2012; 75:1005-1015.
24. Faigo DO. EUS in patients with benign and malignant lymphadenopathy. Gastrointest Endosc. 2001; 53:593598

25. Noemi HA, Antonio ZGG, Juan AOS, Enrique Q. Role of endoscopic ultrasonography with and without fine needle aspiration cytology in the diagnosis and staging of lymphoma. Journal of Hematological Malignancies. 2012; 2:55-60.

26. Palaniappan S, Arvind MA, Dinesh J et al. Role of endoscopic ultrasound-guided fine needle aspiration in the diagnosis of space occupying lesions of the pancreas. Journal of Digestive Endoscopy. 2014; 5:110-115. 